\title{
DELAYED STERNAL CLOSURE AFTER CARDIAC OPERATIONS IN A PEDIATRIC POPULATION
}

\author{
S. Tabbutt, MD, PhD ${ }^{\mathrm{a}, \mathrm{b}, \mathrm{d}, \mathrm{e}}$ \\ B. W. Duncan, $\mathrm{MD}^{\mathrm{c}, \mathrm{f}}$ \\ D. McLaughlin, $\mathrm{RN}^{\mathrm{g}}$ \\ D. L. Wessel, MD a,d $^{\mathrm{a}}$ \\ R. A. Jonas, MD ${ }^{c, f}$ \\ P. C. Laussen, $\mathrm{MB}, \mathrm{BS}^{\mathrm{b}, \mathrm{e}}$
}

\begin{abstract}
Objective: The purpose of this study was to assess morbidity and mortality associated with delayed sternal closure after pediatric cardiac operations. Methods: Hospital records were reviewed of all patients with an open sternum after a cardiac operation at Children's Hospital, Boston, from January 1992 to December 1995. Results: A total of 178 patients had delayed sternal closure with an overall mortality rate of $19 \%$. The most common diagnosis of patients with delayed sternal closure was hypoplastic left heart syndrome $(29 \%)$. Although myocardial distention or chest wall edema $(n=$ 47) was a common indication to delay sternal closure, in many patients $(n=47)$ the sternum was left open electively to avoid postoperative cardiac or respiratory compromise. Successful sternal closure was achieved in $\mathbf{1 5 8}$ patients $(89 \%)$ at a mean of $3.4 \pm 1.8$ days after opening. There were significant increases in left atrial pressure $(7.7 \pm 3.4$ to $9.8 \pm 4.1 \mathrm{~mm} \mathrm{Hg}$, $p=0.00001)$ and right atrial pressure $(8 \pm 3.2$ to $10.1 \pm 3.3 \mathrm{~mm} \mathrm{Hg}, p=$ 0.00001 ) with sternal closure. There was a small but statistically significant drop in $\mathrm{pH}(7.44 \pm 0.05$ to $7.41 \pm 0.08, p<0.0001)$ during sternal closure. The peak inspiratory pressure, delivered breaths per minute, and fraction of inspired oxygen all significantly increased during sternal closure. Clinical evidence of surgical site infection occurred in $12(6.7 \%)$ of the patients with delayed sternal closure; mediastinitis developed in $7(3.9 \%)$ patients. Conclusions: Although delayed sternal closure after complex operations for congenital heart disease is often necessary in the operating room because of edema, unstable hemodynamic conditions, or bleeding, it can also be used electively to aid in hemodynamic and respiratory stability in the initial postoperative period. Our review supports a low morbidity associated with delayed sternal closure in a pediatric population. ( $J$ Thorac Cardiovasc Surg 1997;113:886-93)
\end{abstract}

T he concept of delayed sternal closure (DSC) after adult cardiac operations was described in 1975 when Riahi and associates ${ }^{1}$ reported the use of external traction of the sternum to minimize postoperative tamponade conditions. Gielchinsky and associates $^{2}$ reported the first series of 29 adult patients with DSC in 1981. Subsequent series have

From the Departments of Medicine, ${ }^{a}$ Anesthesia, ${ }^{b}$ and Surgery, ${ }^{c}$ Harvard Medical School, and the Departments of Cardiology, ${ }^{\mathrm{d}}$ Anesthesia, ${ }^{\mathrm{e}}$ Cardiac Surgery, ${ }^{\mathrm{f}}$ and Infection Control, ${ }^{\mathrm{g}}$ Children's Hospital, Boston, Mass.

Received for publication Oct. 11, 1996; revisions requested Nov. 18, 1996; revisions received Dec. 20, 1996; accepted for publication Dec. 31, 1996.

Address for reprints: Sarah Tabbutt, MD, PhD, Department of Anesthesia, MICU, Farley 5, Children's Hospital, 300 Longwood Ave., Boston, MA 02115.

Copyright (C) 1997 by Mosby-Year Book, Inc.

$0022-5223 / 97 \$ 5.00+0 \quad \mathbf{1 2} / \mathbf{1} / \mathbf{8 0 1 0 0}$ reported a $1 \%$ to $2 \%$ incidence of DSC among adult patients undergoing cardiac operations. ${ }^{3-8}$ Reasons for DSC in adults include myocardial edema, bleeding, persistent arrhythmia, and placement of ventricular assist devices.

In 1981, Gangahar, McGough, and Synhorst ${ }^{9}$ reported relief of tamponade conditions after postoperative sternal reopening in an infant. In 1982, Shore, Capuani, and Lincoln ${ }^{10}$ reported a significant decrease in central venous pressure and significant increases in blood pressure and urine output after postoperative sternal reopening done because of low output state. More recently, DSC has been described in a few series after pediatric cardiac operations (Table I).$^{8,10-13}$ The overall mortality rate in pediatric patients undergoing DSC varied from $11 \%$ to $36.2 \%$.

Capillary leak and edema associated with cardiopulmonary bypass continue into the postoperative 
Table I. Summary of pediatric literature

\begin{tabular}{|c|c|c|c|c|c|c|}
\hline Reference & $\begin{array}{c}\text { No. of } \\
\text { patients }\end{array}$ & $\begin{array}{c}\text { Average } \\
\text { age }\end{array}$ & $\begin{array}{c}\text { Incidence of } \\
D S C\end{array}$ & $\begin{array}{c}\text { Mortality } \\
(\%)\end{array}$ & $\begin{array}{c}\text { Superficial } \\
\text { SSI }(\%)\end{array}$ & $\begin{array}{c}\text { Deep } \\
\text { SSI (\%) }\end{array}$ \\
\hline Shore, $1982^{10}$ & 9 & $27 \mathrm{mo}$ & NR & 22 & $0^{*}$ & $0^{*}$ \\
\hline Fanning, $1987^{8}$ & 12 & NR & NR & 17 & 10 & 0 \\
\hline Odim, $1989^{11}$ & 9 & 10 days & $30 \%$ of neonates & 11 & 0 & 0 \\
\hline Hakimi, $1994^{12}$ & 55 & $<30$ days & $62 \%$ of neonates & 20 & 2.4 & 0 \\
\hline Alexi-Meskishuili, $1995^{13}$ & 113 & NR & $9 \%$ & 36 & 0 & 0.9 \\
\hline
\end{tabular}

$S S I$, surgical site infection; $N R$, not reported.

*Incidence among survivors.

period and can compromise myocardial and pulmonary function in infants and children. It is not surprising that DSC is beneficial, and occasionally essential, in the immediate postoperative period. This paper reviews the experience with DSC after cardiac operations at Children's Hospital, Boston, from 1992 to 1995, with a particular focus on the incidence of surgical site infection, the timing of sternal closure relative to daily fluid balance, and the hemodynamic changes associated with sternal closure.

\section{Methods}

Data collection. Hospital records, including operative reports, were reviewed for all patients with DSC after cardiac operations from January 1, 1992, until December 31, 1995, at Children's Hospital, Boston. A total of 239 patients (9\% of 2559 total cases in which bypass was used) had an open sternum in the cardiac intensive care unit (CICU) during this period. However, 22 patients who underwent emergency sternotomies before or after operation in the CICU without DSC were not included in this review. These patients either died $(50 \%)$ within 2 hours (10, arrest; 1 , bleeding) or had successful closure $(50 \%)$ within an hour ( 6 , bleeding; 2 , arrest; 1 , patent ductus arteriosus closure; 1 , systemic pulmonary arterial shunt placement; 1 , pulmonary artery band adjustment). In an additional 39 patients the use of extracorporeal membrane oxygenation $(n=17)$ or ventricular assist devices $(n=22)$ was instituted directly after sternal opening in the $\mathrm{CICU}$ or in the operating room as a transition from bypass. These cases are described elsewhere. ${ }^{14}$ The data for the remaining 178 patients who underwent DSC were reviewed for the present paper.

Factors that contributed to the decision to delay sternal closure after the operation or to open the sternum on an emergency basis in the CICU were noted. These included the patient's preoperative condition, cardiac diagnosis and surgical procedure, duration of bypass, complications related to the operation and bypass, and hemodynamic or respiratory instability.

Surgical technique. At the completion of the operation, sternal wounds were closed with a latex membrane (Esmark Bandage, Critical Specialties, Inc., Westchester, $\mathrm{Pa}$.) sewn to the skin edges with a monofilament suture. The skin edges were covered with a light film of antibiotic ointment (neomycin-polymyxin-bacitracin; Neopoly Ointment, Melville, N.Y.). Fluffed, dry gauze was then applied sterilely and changed daily. Beginning in September 1995 we used a polyurethane, iodine-impregnated, occlusive film (Ioban, 3M Company, Minneapolis, Minn.) over the latex membrane. This dressing was applied once and not changed until final sternal closure or until after an interim procedure that required the chest to be entered. The Ioban dressing allows easy visual identification of fluid accumulation. In four patients only the skin was closed over an open sternum, without use of the Esmark latex membrane. Five patients with severe hemodynamic or respiratory lability had a metal or plastic (cut chest tube or $3 \mathrm{ml}$ syringe) strut placed between the sternal halves.

Medications. All patients received routine perioperative intravenous antibiotic prophylaxis with either cefazolin ( 50 to $75 \mathrm{mg} / \mathrm{kg}$ per day) or vancomycin ( 40 to $60 \mathrm{mg} / \mathrm{kg}$ per day, adjusted to achieve trough and peak levels of $<12$ $\mu \mathrm{g} / \mathrm{dl}$ and 25 to $40 \mu \mathrm{g} / \mathrm{dl}$, respectively) and gentamicin (5 to $7.5 \mathrm{mg} / \mathrm{kg}$ per day, adjusted to achieve trough and peak levels of $<2 \mu \mathrm{g} / \mathrm{dl}$ and 4 to $8 \mu \mathrm{g} / \mathrm{dl}$, respectively). Antibiotic coverage was broadened when there was clinical evidence of mediastinal infection, mediastinal culture results positive for Staphylococcus aureus even in the absence of evidence of clinical infections, or culturepositive infection at other sites. Patients were treated with paralyzing agents (most frequently pancuronium) and heavily sedated with a fentanyl infusion (10 to $15 \mu \mathrm{g} / \mathrm{kg}$ per hour) in the CICU while the sternum was open and during sternal closure. Midazolam $(0.1 \mathrm{mg} / \mathrm{kg}$ per dose $)$ and lorazepam ( $0.1 \mathrm{mg} / \mathrm{kg}$ per dose) were administered as necessary for additional sedation.

Sternal closure. A majority of patients underwent DSC in the CICU under sterile conditions. During closure the mediastinum was explored, thrombus removed, and cultures obtained. The wound was irrigated with a neomycin $(40 \mathrm{mg} / \mathrm{ml})$ and polymyxin $\mathrm{B}(200,000 \mathrm{U} / \mathrm{ml})$ solution. Close monitoring for hemodynamic changes was done and adjustments were made to mechanical ventilation to maintain peripheral arterial oxygen saturation and tidal volume. Preferably, patients had a negative daily fluid balance, decreased body edema, and stable hemodynamic conditions before sternal closure. Nevertheless, sternal closure was usually attempted by postoperative day 7 or 8 . A very small number of critically ill neonates, with prolonged open sternums because of considerable edema, had the sternum closed gradually with additional sternal wires added over a couple days. 
Table II. Annual cases with DSC and associated mortality

\begin{tabular}{|c|c|c|c|c|c|c|c|}
\hline \multirow[b]{2}{*}{ Year } & \multirow[b]{2}{*}{ Bypass cases } & \multicolumn{2}{|c|}{ Total DSC } & \multicolumn{2}{|c|}{ Sternums left open in OR } & \multicolumn{2}{|c|}{ Sternums opened in CICU } \\
\hline & & $n(\%$ total $)$ & Mortality & $n(\%$ total $D S C)$ & Mortality & $n(\%$ total DSC) & Mortality \\
\hline 1992 & 689 & $41(5.9 \%)$ & $6(15 \%)$ & $37(90 \%)$ & $6(16 \%)$ & $4(10 \%)$ & 0 \\
\hline 1993 & 634 & $44(6.9 \%)$ & $8(18 \%)$ & $38(86 \%)$ & $6(16 \%)$ & $6(14 \%)$ & $2(33 \%)$ \\
\hline 1994 & 630 & $57(9.1 \%)$ & $13(23 \%)$ & $50(88 \%)$ & $12(24 \%)$ & $7(12 \%)$ & $1(14 \%)$ \\
\hline 1995 & 606 & $36(6.1 \%)$ & $7(19 \%)$ & $33(92 \%)$ & $7(21 \%)$ & $3(8 \%)$ & 0 \\
\hline Total & 2559 & $178(7 \%)$ & $34(19 \%)$ & $158(89 \%)$ & $31(19 \%)$ & $20(11 \%)$ & $3(15 \%)$ \\
\hline
\end{tabular}

OR, Operating room.

Table III. Characteristics of patients with DSC

\begin{tabular}{lccc}
\hline & Total with DSC & Left open in OR & Opened in CICU \\
\hline No. of patients & 178 & 158 & 20 \\
Patient age at time of operation (days) & $10(0-9312)$ & $9.5(0-9312)$ & $26.5(1-7040)$ \\
Patient weight at time of operation (kg) & $3.4(1.3-69)$ & $3.4(1-3-69)$ & $4(3-57)$ \\
Bypass time (min) & $136(0-504)$ & $136(34-504)$ & $137(0-378)$ \\
Aortic crossclamp time (min) & $60(0-184)$ & $61.5(14-184)$ & $59(0-108)$ \\
Days sternum remained open & $3.4(0-10)$ & $3.5(1-10)$ & $2.4(0-6)$ \\
Postoperative days until extubation & $7(2-77)$ & $7(3-77)$ & $6(2-32)$ \\
Postoperative days until hospital discharge* & $21(6-95)$ & $22(8-95)$ & $17(6-41)$ \\
\hline
\end{tabular}

Data presented are median with range in parentheses. $O R$, Operating room.

* Seven patients were discharged as direct hospital transfers.

Daily fluid balances from the time of the operation until 3 days after sternal closure were calculated on the basis of the patient's preoperative weight. At the time of sternal closure, hemodynamic variables assessed included direct arterial blood pressure, heart rate, and filling pressures obtained from indwelling transthoracic right and left atrial catheters placed at the time of the operation. Atrial pressures in patients with a common atrium, such as after a stage 1 palliation for hypoplastic left heart syndrome (HLHS), were included as right atrial pressures. Arterial blood gas values and mechanical ventilation requirements before and after sternal closure were compared. Additional inotropic requirements during sternal closure were noted. The times to sternal closure, extubation, and hospital discharge were reported.

Culture data. The incidence of surgical site infection was assessed from reported clinical evidence and sternal cultures obtained at the time of sternal closure or subsequently if signs of infection developed. Positive culture findings are maintained in a database in the hospital infection control department. Deep sternal infections, or mediastinitis, were defined as infections that involved the sternum or mediastinum, or both, and were determined by sternal instability, direct inspection during surgical incision and drainage, or positive mediastinal culture result. Superficial infections were defined as infections of the soft tissue and skin proximal to the sternum and determined by erythema with wound dehiscence or drainage, but no evidence of sternal instability.

Statistical analysis. Hemodynamic and respiratory data before and after sternum closure were compared with a two-tailed paired $t$ test. Comparisons between groups of unequal populations were achieved with use of a two-tailed unpaired $t$ test assuming unequal variances or the Wilcoxon rank sum test, or with both tests. Univariate analysis and multivariate logistical regression were used to determine predictors for risk of surgical site infection. A value of $p<0.05$ was considered significant.

\section{Results}

The 178 patients who underwent DSC after cardiac operations from January 1992 through December 1995 represent $7 \%$ of all cases in which cardiopulmonary bypass was used. One hundred fifty-eight of these patients had the sternums left open in the operating room and 20 had the sternums opened in the CICU (Table II). The patient demographics are reported in Table III. There was no significant difference in patient age or bypass time between patients with sternums left open in the operating room and those with sternums opened in the CICU. The overall mortality rate among patients with DSC was $19 \%$ (Table II).

The cardiac diagnoses of the 178 patients with DSC included HLHS and variants thereof (51), transposition of the great arteries (TGA) with or without associated lesions (42), tetralogy of Fallot (18), truncus arteriosus (9), ventricular septal defect including double-outlet right ventricle (9), total anomalous pulmonary venous return with obstruction (5), single ventricle (other than HLHS) neces- 
sitating stage 1 palliation (4), single ventricle needing bidirectional Glenn operation and correction of associated lesions (6), single ventricle needing Fontan operation (6), interrupted aortic arch with ventricular septal defect (5), complex lesions necessitating systemic pulmonary arterial shunt palliations (3), valve anomalies necessitating valve replacement (4), complete atrioventricular canal (4), and other (12).

Patients who underwent a stage 1 palliation for HLHS and variants thereof comprised $29 \%$ of all patients with DSC in the operating room, and patients with TGA who underwent an arterial switch procedure with or without repair of associated lesions comprised $24 \%$. During the study period, $49 \%$ (51/104) of all patients with HLHS and $22 \%$ (42/ 194) of patients with TGA underwent DSC.

The reasons for leaving the sternum open in the operating room, obtained from the operative note, are shown in Table IV. Myocardial distension or chest wall edema ( $n=47$ ) was a common indication. However, 47 of the sternums were left open electively to avoid postoperative cardiac or respiratory compromise. Of the patients whose sternums were left open electively, 52\% had HLHS being treated with stage 1 palliation and $17 \%$ had TGA with or without associated lesions. Very few patients required sternal reopening in the operating room after surgical closure. Bleeding (30\%) and unstable hemodynamic conditions $(30 \%)$ were the most common reasons for sternal opening and subsequent DSC in the CICU (Table IV).

Successful sternal closure was achieved in 158 patients $(89 \%)$ at a mean of $3.4 \pm 1.8$ days after opening (Table III). Fourteen patients subsequently died a median of 22 days (range 1 to 106 days) after sternal closure. Twenty patients $(11 \%)$ died before sternal closure. Because of hemodynamic instability, there were 14 failed attempts at sternal closure among 12 patients at a mean of 3 days after the operation. Successful closure was accomplished in all of these patients a median of 2 days (range 1 to 5 days) after the failed attempt or attempts. No deaths occurred the same day as sternal closure or attempted sternal closure. Patients with failed attempts at sternal closure did not differ significantly from those with successful closures in age, weight, bypass time, fluid balance, or right atrial pressure. The number of days until sternal closure did not correlate with patient age (correlation coefficient, $r=-0.15$ ), the cardiopulmonary bypass time (correlation coefficient, $r=0.18$ ), or the aortic cross-
Table IV. Indications for opening sternum in $C I C U$ and decision to delay sternal closure in operating room

\begin{tabular}{|c|c|c|c|c|}
\hline & \multicolumn{2}{|c|}{$\begin{array}{l}\text { Indication } \\
\text { for opening } \\
\text { sternum in } \\
\quad \text { CICU }\end{array}$} & \multicolumn{2}{|c|}{$\begin{array}{l}\text { Indication } \\
\text { for leaving } \\
\text { sternum } \\
\text { open in } O R\end{array}$} \\
\hline & $n$ & $\%$ & $n$ & $\%$ \\
\hline Arrest & 5 & 25 & & \\
\hline Procedure* & 2 & 10 & & \\
\hline Bleeding & 6 & 30 & 9 & 6 \\
\hline $\begin{array}{l}\text { Unstable hemodynamic } \\
\text { conditions }\end{array}$ & 6 & 30 & 26 & 16 \\
\hline Electivet & & & 47 & 30 \\
\hline Edema & & & 47 & 30 \\
\hline Premature infant & & & 4 & 2.5 \\
\hline Hypoxia & & & 8 & 5 \\
\hline Arrhythmia & & & 1 & 0.5 \\
\hline Long bypass time & & & 8 & 5 \\
\hline Unknown & 1 & 5 & 8 & 5 \\
\hline
\end{tabular}

$O R$, Operating room.

*Procedures included placing intracardiac lines, adjusting systemic pulmonary arterial shunts, and closing a patent ductus arteriosus.

$\dagger$ Reflects operative notes that either specifically state sternum was left open electively or there was no other specific cause for DSC.

clamp time (correlation coefficient, $r=0.16$ ). Before sternal closure, mediastinal exploration was necessary on 61 occasions in 50 patients because of cardiac arrest $(n=15)$, bleeding $(n=24)$, intrathoracic procedures $(n=12)$, and hypotension $(n=$ $10)$.

Fluid balance data were available for 120 of the 158 patients whose sternums were closed (Fig. 1). On the average, patients had a negative fluid balance for 3 days before sternal closure. In general, patients with an uncomplicated postoperative course had a positive fluid balance the first postoperative night, followed by a negative fluid balance on subsequent days until sternal closure.

Hemodynamic and respiratory data were available for 121 patients who underwent sternal closure (Table V). There were significant increases in left and right atrial pressures, systolic and diastolic blood pressure, and heart rate with sternal closure. Inotropic requirements in 117 patients were increased in $24 \%$, decreased in $8.5 \%$, and unchanged in $67.5 \%$ of patients during sternal closure.

Although there was a small, but statistically significant drop in $\mathrm{pH}$, arterial oxygen tension and carbon dioxide tension values remained essentially unchanged during sternal closure, reflecting simultaneous changes in mechanical ventilation to maintain a stable minute ventilation. The values of peak 




Fig. 1. Postoperative daily fluid balance. Data presented are mean plus or minus $95 \%$ confidence intervals.

Table V. Hemodynamic and respiratory variables during sternal closure

\begin{tabular}{|c|c|c|c|c|}
\hline & Before sternal closure & After sternal closure & $p$ Value & $n$ \\
\hline $\mathrm{LAP}(\mathrm{mm} \mathrm{Hg})$ & $7.7 \pm 3.4$ & $9.8 \pm 4.1$ & 0.00001 & 46 \\
\hline $\mathrm{RAP}(\mathrm{mm} \mathrm{Hg})$ & $8 \pm 3.2$ & $10.1 \pm 3.3$ & 0.00001 & 108 \\
\hline $\mathrm{SBP}(\mathrm{mm} \mathrm{Hg})$ & $73.1 \pm 15$ & $75.6 \pm 16$ & 0.04 & 121 \\
\hline $\mathrm{DBP}(\mathrm{mm} \mathrm{Hg})$ & $41.5 \pm 10$ & $44.6 \pm 11$ & 0.0003 & 121 \\
\hline Heart rate (beats/min) & $146.4 \pm 18$ & $161.4 \pm 18$ & 0.00001 & 69 \\
\hline $\mathrm{pH}$ & $7.44 \pm 0.05$ & $7.41 \pm 0.08$ & 0.00001 & 119 \\
\hline $\mathrm{PCO}_{2}(\mathrm{~mm} \mathrm{Hg})$ & $45.2 \pm 7.8$ & $46.3 \pm 9.7$ & 0.16 & 119 \\
\hline $\mathrm{PO}_{2}(\mathrm{~mm} \mathrm{Hg})$ & $79.7 \pm 43$ & $79.0 \pm 49$ & 0.80 & 119 \\
\hline $\mathrm{PIP}\left(\mathrm{cm} \mathrm{H}_{2} \mathrm{O}\right)$ & $27.1 \pm 4$ & $28.6 \pm 4$ & 0.00001 & 115 \\
\hline PEEP $\left(\mathrm{cm} \mathrm{H}_{2} \mathrm{O}\right)$ & $4.2 \pm 1$ & $4.2 \pm 1$ & 0.42 & 114 \\
\hline IMV (breaths/min) & $13.5 \pm 3.6$ & $14.8 \pm 4.2$ & 0.00001 & 116 \\
\hline $\mathrm{FiO}_{2}(\%)$ & $33.9 \pm 12$ & $39.9 \pm 19$ & 0.00004 & 118 \\
\hline Tidal volume $(\mathrm{ml} / \mathrm{kg})$ & $16.5 \pm 7.6$ & $16.2 \pm 7.5$ & 0.42 & 86 \\
\hline
\end{tabular}

Data presented as mean plus or minus the standard deviation. $n$, Number of patients for whom data were available; $L A P$, left atrial pressure measured by intracardiac line; $R A P$, right atrial or common atrial pressure measured by intracardiac line; $S B P$, systolic blood pressure; $D B P$, diastolic blood pressure; $P C C_{2}$, carbon dioxide tension; $P_{2}$, oxygen tension; $P I P$, peak inspiratory pressure, $P E E P$, peak end-expiratory pressure; $I M V$, intermittent mandatory ventilation; $\mathrm{FiO}_{2}$, fractional inspired oxygen; Tidal volume, tidal volume in milliliters per kilogram preoperative weight.

inspiratory pressure, delivered breaths per minute, and fraction of inspired oxygen all significantly increased during sternal closure.

Clinical evidence of surgical site infection occurred in $12(6.7 \%)$ of the patients with DSC (Table VI). Seven patients had a deep surgical site infection, or mediastinitis, with an unstable sternum necessitating incision and drainage in the operating room (median postoperative day 24, range 19 to 33 days). Mediastinal cultures grew Staphylococcus aureus in four patients and coagulase-negative Staphylococcus in one patient and were negative in two patients. Five patients had a superficial surgical site infection; three had positive culture results for $S$. aureus, one had a finding of coagulase-negative Staphylococcus, and one had culture negative results. One patient with a superficial surgical site infection (coagulase-negative Staphylococcus) and three patients with mediastinitis ( $S$. aureus) had concurrent blood cultures positive for the same organism. All patients received intravenous antibiotic therapy and had no recurrence of the infection. In one patient, whose sternum was closed on postoperative day 1 , sepsis unresponsive to antibiotics developed and the patient died on postoperative day 11. Blood and mediastinal cultures at the time of 
Table VI. Characteristics of patients with surgical site infections

\begin{tabular}{lccc}
\hline & No infection & Superficial SSI & Deep SSI \\
\hline No. of patients & $166(93.3 \%)$ & $5(2.8 \%)$ & $7(3.9 \%)$ \\
Patient age (days) & $10(0-9312)$ & $3(2-17)^{\%}$ & $14(2-24)$ \\
Patient weight (kg) & $3.4(1.3-69)$ & $3.4(3-4)$ & $3.4(3-4.5)$ \\
Bypass time (min) & $132(0-504)$ & $209(197-210) \dagger$ & $140(111-320)$ \\
Sternum open (days) & $3(0-10)$ & $3(2-5)$ & $3(1-9)$ \\
Sternal reexploration & $47(28 \%)$ & $1(20 \%)$ & $2(29 \%)$ \\
\hline
\end{tabular}

Data presented as median with range in parentheses or as number and percent. Statistical significance determined by the Wilcoxon rank sum test. SSI, Surgical site infection.

${ }^{*} p=0.06$.

$\downarrow p=0.077$.

death were positive for $S$. aureus. One patient with culture-negative mediastinitis and one patient with $S$. aureus mediastinitis died with acute renal failure on postoperative days 26 and 30 , respectively. One patient with a superficial surgical site infection culture positive for coagulase-negative Staphylococcus required bilateral pectoralis advancements before hospital discharge.

Univariate and multivariate analyses found no significant predictors for risk of surgical site infection among bypass time, time the sternum remained open, or number of postoperative sternal explorations. Although patients with infection were younger, this was not significant. Of the patients with mediastinitis, $43 \%$ had HLHS or variants thereof and underwent a stage 1 palliation and $43 \%$ had TGA with or without associated lesions.

\section{Discussion}

Pericardial and sternal closure after cardiac operations produce a constrictive effect on cardiac func$\operatorname{tion}^{10,15-17}$ and may interfere with efficient mechanical ventilation. This is important in infants in whom considerable capillary leak and edema may develop after cardiopulmonary bypass and in whom cardiopulmonary interactions have a significant impact on immediate postoperative recovery. DSC provides significant, and occasionally essential, hemodynamic and pulmonary stability. It is important to evaluate the potential morbidity associated with DSC, particularly if it is used in an elective manner for neonates and children at higher risk as a means of maintaining hemodynamic and respiratory stability in the initial postoperative period.

Our retrospective review is the largest reported series of pediatric patients undergoing DSC after operations for congenital cardiac diseases. Our incidence of DSC was $7 \%$ of all patients having cardiac operations with cardiopulomary bypass and demonstrates a low morbidity for surgical site infections and cardiorespiratory instability during sternal closure. Related to the low morbidity, $30 \%$ of the cases of sternums left open in the operating room were done so electively by the surgeon in anticipation of hemodynamic and respiratory instability in the initial postoperative period. In these patients age (median 5 days, range 1 to 9312 days), weight (3.3 kg, range 2.2 to $69 \mathrm{~kg}$ ), and mortality (20\%) were similar to those of the overall DSC population. Of the patients whose sternums were left open electively in the operating room, $52 \%$ had undergone stage 1 palliation for HLHS. In this group of patients, the balance between pulmonary and systemic blood flow is critical.

Our mortality rate of $19 \%$ for patients with DSC is comparable to the literature range of $11 \%$ to $36 \% ., 10-13$ These patients represent a surgical population at higher risk. Patients with DSC had a median weight of $3.4 \mathrm{~kg}$. Many of the patients had hemodynamically compromised conditions before operation. Of the patients whose sternums were left open in the operating room, $74 \%$ were younger than 1 month old and $31 \%$ had stage 1 palliation for HLHS and variants thereof. Eighty-five percent of the sternums open in the CICU were left open because of unstable hemodynamic conditions, cardiac arrest, or bleeding.

Large series in adult patients have reported the incidence of wound infection after heart operations at $1.5 \%$ to $1.8 \%{ }^{18,19}$ The pediatric literature reports an incidence of sternal wound infection after cardiac operations (with and without cardiopulmonary bypass) from $0.1 \%$ to $7 \%, 21$ and an incidence of $0 \%$ to $28 \%$ in patients with DSC. ${ }^{8,10-13,20}$ We found a $6.7 \%$ incidence of surgical site infection and a $3.9 \%$ incidence of mediastinitis in our patients with DSC.

Although all attempts were made to maintain a sterile field when the sternum was opened after the 
operation in the CICU, in an emergency situation the surgical barrier may have been broken. The incidence of surgical site infection was $10 \%$ for patients in whom the sternum was opened in the CICU (5\% mediastinitis) compared with $6.2 \%$ for patients in whom the sternum was left open in the operating room (3.7\% mediastinitis).

Pollock and colleagues ${ }^{20}$ found the risk of sternal wound infection for all patients after pediatric cardiac operations to be higher if the patient had an open sternum in the CICU ( $27.6 \%$ had development of sternal wound infection), if the patient had a higher severity of illness at the time of postoperative admission to the ICU (10.3\% had development of sternal wound infection), and for certain cardiac diagnoses (double-outlet right ventricle, truncus, TGA, and valvotomy being most common). Edwards and Baker ${ }^{21}$ found risk factors for sternal wound infections in children after cardiac operations included pump bypass time longer than 1 hour, excessive postoperative bleeding, extended low cardiac output after operation, and inadequate antimicrobial prophylaxis. They did not specifically address cases of patients with open sternums. We found no statistical differences in the incidence of mediastinitis (deep surgical site infection) between length of cardiopulmonary bypass, number of postoperative days until sternal closure, or frequency of sternal reexploration in the CICU. However, patients with superficial wound infections or mediastinitis were statistically younger. Most of our patients with mediastinitis had HLHS $(43 \%, n=3)$ or TGA with or without associated lesions $(43 \%, n=3)$.

The choice and duration of antibiotic prophylaxis may influence the risk for surgical site infection in patients with DSC. Pollock and colleagues ${ }^{20}$ used gentamicin until sternums were closed with mediastinitis developing in $10.3 \%(3 / 29)$ of patients. A recent series used triple antibiotic coverage with cefotaxime, piperacillin, and teicoplanin (glycopeptide) until 3 days after sternal closure and reported a $0.9 \%(1 / 113)$ incidence of mediastinitis. ${ }^{13}$ Hakimi and associates ${ }^{12}$ used a first-generation cephalosporin until sternal closure followed by penicillinaseresistant penicillin and an aminoglycoside and reported no cases $(0 / 55)$ of mediastinitis. We used cephazolin (50 to $75 \mathrm{mg} / \mathrm{kg}$ per day) or vancomycin (40 to $60 \mathrm{mg} / \mathrm{kg}$ per day) and gentamicin (5 to 7.5 $\mathrm{mg} / \mathrm{kg}$ per day) until sternal closure, with additional coverage if culture-positive infection developed at other sites. We observed a $3.9 \%(7 / 178)$ incidence of mediastinitis.
The establishment of diuresis and negative fluid balance has been correlated with successful sternal closure in one adult series. ${ }^{7}$ This is supported in our review because most patients had achieved a negative fluid balance for 3 days before sternal closure. We view this as an important indicator for achievement of successful sternal closure. Immediately after cardiac operations our patients are restricted to $50 \%$ maintenance fluids and administration of diuretics is routinely initiated on postoperative day 1 to promote a negative fluid balance.

Hemodynamic changes are common at the time of sternal closure. Increases in atrial pressure, blood pressure (primarily diastolic), and heart rate were well tolerated. Careful monitoring is essential at the time of sternal closure. Among our patients, 12 had failed initial closure attempts as a result of hemodynamic instability; subsequent closure was successfully achieved. Failure did not reflect a significant difference in fluid balance, bypass time, diagnosis, age, weight, or atrial pressure.

Mechanical ventilation requirements increase with sternal closure. Significant increases in fraction of inspired oxygen, peak inspiratory pressure, and ventilatory rate were required to maintain tidal volume and gas exchange. The increase in ventilatory requirement may contribute to the increase in atrial pressure.

Although DSC is often necessary in the operating room because of edema, bleeding, or unstable hemodynamic conditions, it can also be considered an elective option to aid in hemodynamic and respiratory stability in the initial postoperative period. After a sustained negative fluid balance is achieved, sternums can be successfully closed at a mean of 3.4 postoperative days. The increased mortality in this group of patients most likely reflects the higher surgical risk associated with the diseases present. Although DSC does not appear to be associated with an increased morbidity, a prospective comparison with conventional closure is not possible because many of these patients have no option except DSC. DSC has become an important adjunct to complex cardiac operations in neonates and children.

We thank Kimberlee Gauvreau, ScD, for statistical assistance.

\section{REFERENCES}

1. Riahi M, Tomatis LA, Schlosser RJ, Bertolozzi E, Johnston DW. Cardiac compression due to closure of the median sternotomy in open heart surgery. Chest 1975;67:113-4.

2. Gielchinsky I, Parsonnet V, Krishnan B, Silidker M, Abel 
RM. Delayed sternal closure following open-heart operation. Ann Thorac Surg 1981;32:273-7.

3. Johnson JA, Gundersen AE, Stickney ID, Cogbill TH. Selective approach to sternal closure after exploration for hemorrhage following coronary artery bypass. Ann Thorac Surg 1990;49:771-4

4. Milgater E, Uretzky G, Shimon DV, Silberman S, Appelbaum A, Borman JB. Delayed sternal closure following cardiac operations. J Cardiovasc Surg 1986;27:328-31.

5. Josa M, Khuri SF, Braunwald NS, et al. Delayed sternal closure. J Thorac Cardiovasc Surg 1986;91:598-603.

6. Mestres CA, Pomar JL, Acosta M, et al. Delayed sternal closure for life-threatening complications in cardiac operations: an update. Ann Thorac Surg 1991;51:773-6.

7. Furnary AP, Magovern JA, Simpson KA, Magovern GJ. Prolonged open sternotomy and delayed sternal closure after cardiac operations. Ann Thorac Surg 1992;54:233-9.

8. Fanning WJ, Vasko JS, Kilman JW. Delayed sternal closure after cardiac surgery. Ann Thorac Surg 1987;44:169-72.

9. Gangahar DM, McGough EC, Synhorst D. Secondary sternal closure: a method of preventing cardiac compression. Ann Thorac Surg 1981;31:281-2.

10. Shore DF, Capuani A, Lincoln C. Atypical tamponade after cardiac operation in infants and children. J Thorac Cardiovasc Surg 1982;83:449-52.

11. Odim JNK, Tchervenkov CI, Dobell ARC. Delayed sternal closure: a lifesaving maneuver after early operation for complex congenital heart disease in the neonate. $\mathrm{J}$ Thorac Cardiovasc Surg 1989;98:413-6.

12. Hakimi M, Walters HL, Pinsky WW, Gallagher MJ, Lyons
JM. Delayed sternal closure after neonatal cardiac operations. J Thorac Cardiovasc Surg 1994;107:925-33.

13. Alexi-Meskishvili V, Weng $Y$, Uhlemann F, Lange PE, Hetzer R. Prolonged open sternotomy after pediatric open heart operation: experience with 113 patients. Ann Thorac Surg 1995;59:379-83.

14. Duncan BW, Hraska V, Jonas RA, et al. Mechanical circulatory support for pediatric cardiac patients [abstract]. Circulation 1996;94(Suppl):I173.

15. Jogi $P$, Werner $\mathrm{O}$. Hemodynamic effects of sternum closure after open-heart surgery in infants and children. Scand J Thorac Cardiovasc Surg 1985;19:217-20.

16. Daughters GT, Frist WH, Alderman EL, Derby GC, Ingels NB, Miller DC. Effects of the pericardium on left ventricular diastolic filling and systolic performance early after cardiac operations. J Thorac Cardiovase Surg 1992;4:1084-91.

17. Hanley FL. The influence of paracardiac structures and conditions on cardiac performance in the postcardiotomy period [Invited letter]. J Thorac Cardiovasc Surg 1992;104:1167-78.

18. Culliford AT, Cunningham JN, Zeff RH, Isom OW, Teiko $P$, Spencer FC. Sternal and costochondral infections following open-heart surgery. J Thorac Cardiovasc Surg 1976;72:714-26.

19. Serry C, Bleck PC, Javid H, et al. Sternal wound complications. J Thorac Cardiovasc Surg 1980;80:861-7.

20. Pollock EMM, Ford-Jones EL, Rebeyka I, et al. Early nosocomial infections in pediatric cardiovascular surgery patients. Crit Care Med 1990;18:378-84.

21. Edwards MS, Baker CJ. Median sternotomy wound infections in children. Pediatr Infect Dis 1983;2:105-9.

\section{Availability of Journal back issues}

As a service to our subscribers, copies of back issues of The Journal of Thoracic and Cardiovascular Surgery for the preceding 5 years are maintained and are available for purchase from Mosby at a cost of $\$ 15.00$ per issue until inventory is depleted. The following quantity discounts are available: $25 \%$ off on quantities of 12 to 23 , and one third off on quantities of 24 or more. Please write to Mosby-Year Book, Inc., Subscription Services, 11830 Westline Industrial Drive, St. Louis MO 63146-3318, or call 800-453-4351 or 314-453-4351 for information on availability of particular issues. If unavailable from the publisher, photocopies of complete issues may be purchased from UMI, 300 N. Zeeb Rd., Ann Arbor, MI 48106, 313-761-4700. 